Special Issue: Biological Effects of Space Radiation -Part II-

\section{Introduction to The Proposed Space Experiments Aboard The ISS Using The Silkworm, Bombyx mori}

\author{
Toshiharu Furusawa ${ }^{1 \dagger}$, Kumie Nojima ${ }^{2}$, \\ Masatoshi Ichida ${ }^{1}$, Sumiharu Nagaoka ${ }^{3}$, Yukio \\ Sugimura $^{3}$, Eiko Suzuki', Motoyuki Sumida', \\ Hiromi Suzuki ${ }^{4,5}$, Toru Simazu ${ }^{5}$, Katsunori \\ Omori $^{6}$, Noriaki Ishioka ${ }^{4,6}$, Hiroshi Fujii ${ }^{7}$, Shunji \\ Nagaoka ${ }^{8}$
}

${ }^{1}$ Center for Bioresource Field Science, Kyoto Institute of Technology, Ipponki-chou, Ukyou-ku, 6168354 Kyoto, Japan

${ }^{2}$ National Institute of Radiological Sciences, Inage, Chiba 263-8555, Japan

${ }^{3}$ Department of Applied Biology, Kyoto Institute of Technology, Matsugasaki, Sakyo-ku, Kyoto 6068585, Japan

${ }^{4}$ Department of Space Environmental Medicine, Graduate School of Medical and Dental Science, Kagoshima University, 8-35-1 Sakuragaoka, Kagoshima-shi, Kagoshima 890-8544, Japan ${ }^{5}$ Technology and Research Promotion Department, Japan Space Forum, Shin-Otemachi BIdg. 7F, 2-21 Otemachi, Chiyoda-ku, Tokyo 100-0004, Japan. ${ }^{6}$ Japan Aerospace Exploration Agency, Tsukuba, Ibaraki 305-8505, Japan

${ }^{7}$ Institute of Genetic Resources, Kyushu University, Hakozaki Higashi-ku Fukuoka 812-8581, Japan

${ }^{8}$ School of Health Science, Fujita Health University, Toyoake, Aichi 470-110, Japan

\section{Abstract}

The authors have a plan to examine the biological effects of cosmic rays by loading the eggs of the silkworm, Bombyx mori, in the International Space Station (ISS) for 3 months. In advance of the project, several ground experiments have been performed. In order to investigate the biological effects of radiation, heavy ion particles were used instead of cosmic rays. Heterozygous eggs of the black-striped strain $\left(p^{s} / p\right)$ were irradiated with Carbon (C), Neon (Ne) or Ferrous (Fe) ion particles. At the fifth instar stage, larvae that hatched from these eggs showed white spots on their backs against the black or dark brown of their integument. These are somatic mutations which seem to be caused by the effects of radiation on the $p^{s}$ gene. The incidence of this somatic mutation increased in proportion to dose and linear energy transfer of $\mathrm{C}$ and $\mathrm{Ne}$ ion particles,

Received: April 19, 2009; Accepted: July 25, 2009

†To whom correspondence should be addressed:

Tel.: +81-(0)75461-5940; E-mail: zai-kinugasakai@nifty.com and it was higher after resumption of embryogenesis in eggs that had been irradiated after diapause termination as compared with eggs irradiated while still in diapause. Irradiation by more than 0.04 Gy of $\mathrm{Fe}$ ion particles to diapause-terminated eggs induced a significant incidence of somatic mutation compared with controls $(P<0.05)$. Furthermore, radiation effects were also detected at the next generation as detected by egg color mutations by using the specific locus method. In experiments investigating the effects of microgravity on silkworm embryogenesis in the US Space Shuttle/Atlantis (STS-84) in 1997, we had observed that microgravity could influence embryonic reversal, presumably resulting in abnormal development of embryos. On the basis of the results from STS-84 flight and the above mentioned ground experiments, the authors will examine the interrelationship between the dose of cosmic rays and the incidence of somatic mutation, as well as confirm and extend the analysis of synergistic effects between cosmic rays and microgravity on mutation rates in experiments conducted using the ISS. These data will provide fundamental information on the effects of cosmic rays on biological systems that can then be applied to better protect humans against cosmic radiation during space flight. (02009 Jpn. Soc. Biol. Sci. Space; Article ID: 092302007

Key Words: Somatic mutation, Heavy ion particle, Silkworm egg, Bomby mori, Black-striped strain

\section{Introduction}

The International Space Station (ISS) is orbiting the Earth at a height of over $400 \mathrm{~km}$. There, its environmental conditions include microgravity and radiation from cosmic rays and heavy ion beams which come from solar flares of our sun and from other parts of the galaxy. Astronauts who stay in the ISS are always exposed to cosmic radiation, and thus a biodosimetric assessment of health risks associated with radiation exposure has been requested. In order to develop biodosimetry, the responses of living organisms to the space environment need to be examined. To date, several kinds of organisms have been analyzed by loading them into flights of the space shuttle; these provide relatively short exposures to cosmic radiation due to short flight times. Experiments using insects have shown the effects of cosmic radiation and microgravity on the larvae of Drosophila melanogaster (Inoguchi and Ikenaga, 2000), and embryos of Carasius morosus (Bucker et al., 1986). These experiments showed that a high frequency of mutation occurred in flight samples when compared with controls maintained on earth, and also suggested that there is a synergistic interaction of high energy heavy (HZE) particle hits and microgravity. Similarly, the biological effects of cosmic radiation on insects could be enhanced under microgravity, but any effects of space radiation, microgravity, or both were not detectable in the frequencies of micronuclei of human, mouse or hamster cell lines that were grown in a 9-day mission (Ikenaga et al., 2002). 


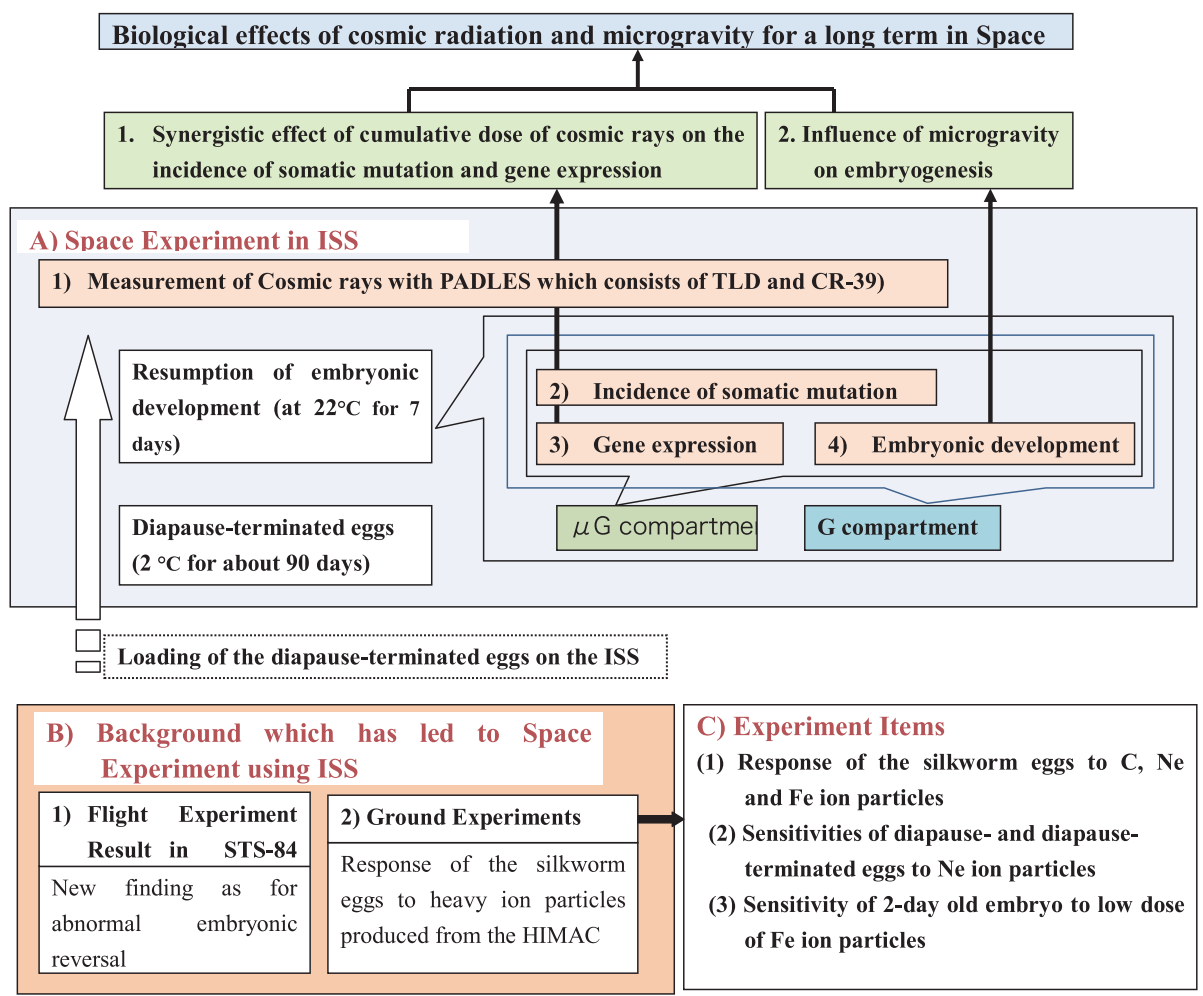

Fig. 1. Experimental items in the Space Experiment in the International Space Station (ISS) (Frame A) and the background studies (Frame B) that led to the Space Experiment. The background work consisted of 1) results from flight experiments aboard the US Space Shuttle/Atlantis (STS-84) in 1997, and 2) ground experiments which consisted of three experimental items (Frame C).

PADLES: Passive Dosimeter for Life Science Experiments in Space, TLD: Thermoluminescent dosimeter, CR-39: Cosmic ray detector film. HIMAC: Heavy Ion Medical Accelerator in Chiba of National Institute of Radiological Sciences

In 1997, silkworm eggs of a mutant strain (extracrescent and degenerated abdominal legs, $\left.E^{D \ell}\right)$ were loaded on the US Space Shuttle Atlantis (STS-84) for a 9 day flight as a test animal (Furusawa et al., 1998). This strain has E-pseudo allelic genes (Chromosome VI, locus 0.0) which belong to a homeotic mutant and are expressed during early cell differentiation of the embryo, leading to a morphological disorder such as fusion of the $4^{\text {th }}$ and $5^{\text {th }}$ segments. The appearance of this abnormality as well as the appearance of star marking and abnormal crescent marking in the flight sample was significantly higher than that seen among ground controls. It is likely that the higher incidence of abnormality in the flight sample was caused by abnormal dorsal closure (Shimada et al., 1986) under microgravity rather than the influence of cosmic rays. This is because there was no significant difference in the absorbed dose of radiation between normal and abnormal larvae which were hatched from eggs of the flight sample, and also we observed that embryonic reversal could not proceed under microgravity (Furusawa et al., 1998). However, in diapausing eggs of the silkworm that were loaded onto the Russian Space Station Mir for about 40 days, the incidence of egg color mutation in their first filial generation was $12.4 \times 10^{-3}$ in the flight sample compared with $10^{-5}$ in the ground control (Morimoto and Sakai, 1998). These results show that silkworm eggs will be a useful model animal in which to examine the biological effects of cosmic rays and microgravity. The model is especially useful because we can regulate the egg-diapause period in the life cycle of the silkworm, Bombyx mori, by controlling the preservation temperature of the eggs. In addition, the phenomenon of embryonic reversal and the expression of specific genes, such as trehalase (Huang et al., 2005) and NAD-sorbitol dehydrogenase (Sakano et al., 2004), are highly specific to different stages of embryonic development.

Accordingly, we have been asked to examine the biological effects of cosmic radiation and microgravity during long term space flight as shown in Figure 1. The first experiment is to investigate the synergistic effects of a cumulative dose of cosmic rays on the incidence of somatic mutation and gene expression, and the second one is to investigate the influence of microgravity on embryogenesis.

In this article, the authors introduce the "Space Experiment in the International Space Station (ISS)" (Fig. $1 \mathrm{~A}$ ), and subsequently the "Background which led to the Space Experiment using the ISS".

\section{Space Experiment using the ISS}

In order to clarify the biological effects of cosmic rays and microgravity using Bombyx eggs, our research will 


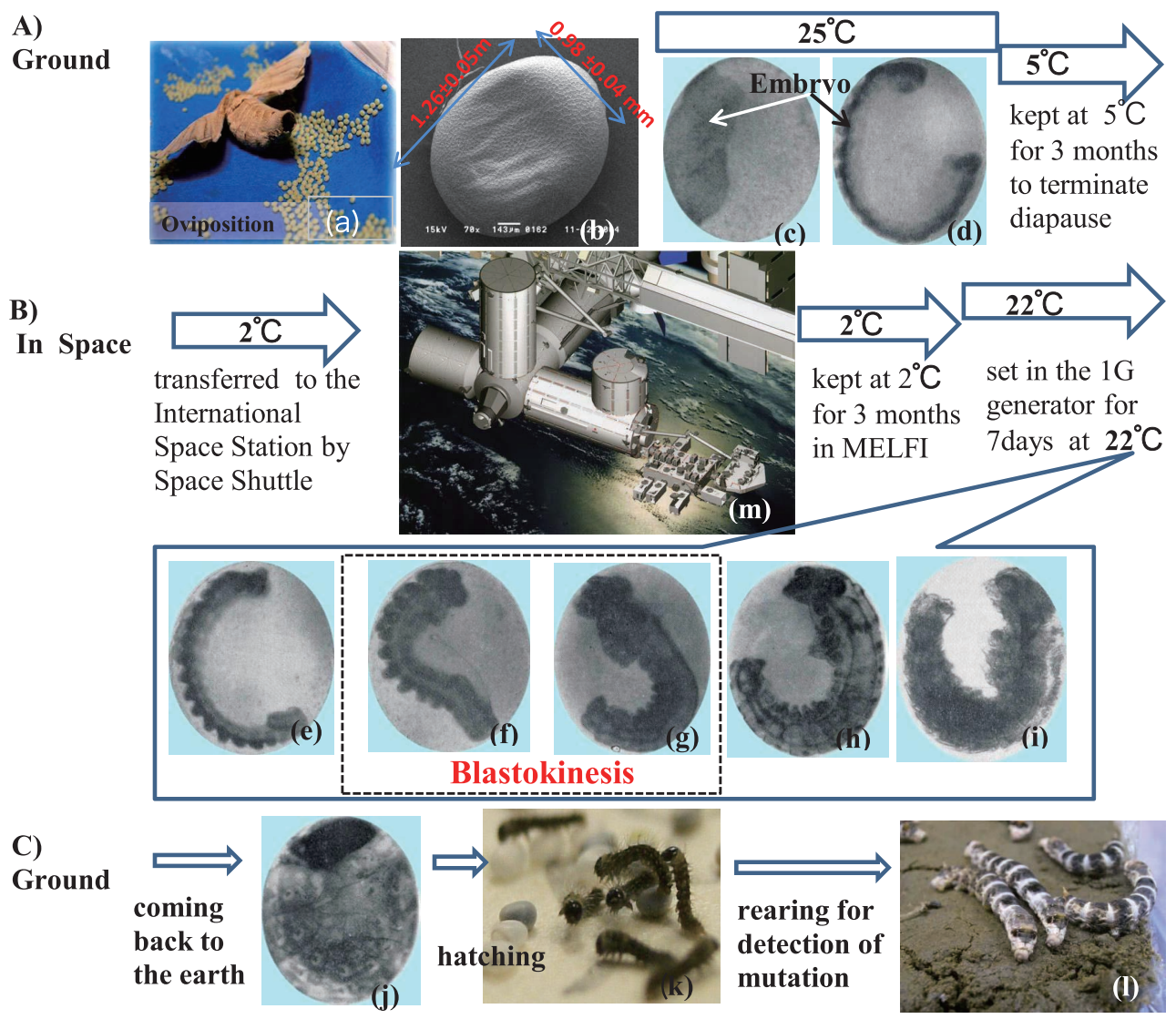

Fig. 2. Development of embryos in eggs of the silkworm, Bombyx mori, from the preparation of sample eggs, to the exposure of these eggs to cosmic radiation in the Japanese Experiment Module, and to the return to Earth. After oviposition (a) eggs with a size of $1.3 \times 1.0 \mathrm{~mm}$ (b), undergo embryonic development for 2 or 3 days (c and d) and then enter into the diapause state (d). If the eggs are kept continuously at a warm ambient temperature $\left(\right.$ e.g. $\left.25^{\circ} \mathrm{C}\right)$, the embryos will die 3 or 4 months later. However, when the eggs are kept in the cold at $5^{\circ} \mathrm{C}$, they survive and terminate diapause after about 3 months. These diapause-terminated eggs are then transferred to the Japanese Experiment Module (JEM) $(\mathrm{m})$, and held there at $2^{\circ} \mathrm{C}$ for 3 months in the Minus Eighty Degree Celsius Laboratory Freezer for the ISS (MELFI). Thereafter, groups of these eggs are transferred to both the $\mu \mathrm{G}$ compartment and the $\mathrm{G}$ compartment for a Gravity Contrast Experiment in the Cell Biology Experiment Facility (CBEF); there they are held at $22^{\circ} \mathrm{C}$ for 7 days. During this period embryogenesis resumes $(e-i)$; blastokinesis or embryonic reversal occurs about 2 days after the resumption. Before hatching $(\mathrm{k})$, the eggs $(\mathrm{j})$ are returned under cold conditions to the Earth, and are then reared to check the incidence of mutation at the fifth instar of the black-striped strain (I). The photos of the embryos are modified from Takami and Kitazawa (1960). c) head-lobe differentiation, d) abdominal outgrowth appearance, e) head and thorax differentiation, f) middle stage of embryonic reversal, g) late stage of embryonic reversal, h) tubercle appearance, i) tenidium formation, j) abdominal pigmentation one day before hatching.

be undertaken according to the following experimental items as shown in Fig.1A; 1) measurement of cosmic rays during the loading of the silkworm eggs with the Passive Dosimeter for Life Science Experiments in Space (PADLES) which consists of a thermoluminescent dosimeter (TLD) and cosmic ray detector film (CR-39). 2) incidence of somatic mutation caused by irradiation of the silkworm eggs by cosmic rays, 3) gene expression during embryogenesis, and 4) embryonic development under microgravity. Item numbers 2-4 will be performed in the $\mu G$ and $G$ compartments in the Cell Biology Experiment Facility (CBEF) of the ISS. In the G compartment, gravity can be controlled from 0.1 to $2.0 \mathrm{G}$.

The silkworm life cycle and the animal experimental protocol that will be used in these experiments are described in Fig. 2. Silkworm larvae are reared on mulberry leaves or an artificial diet, and then produce many eggs (about 200 300 eggs/female moth). In these tiny eggs (about $1.3 \times 1 \mathrm{~mm}$ in size), embryos develop for about two days, but then stop cell division, arresting embryonic development and causing the embryos to enter a diapause state. Diapause cannot be broken until the embryos experience a prolonged period of cold exposure. In the lab, embryos held at $5^{\circ} \mathrm{C}$ for 2-3 months are then able to terminate diapause (Fig. 2A). These diapause-terminated eggs will be transferred under cold conditions by a Space Shuttle to the ISS, and will be exposed there to cosmic radiation for 3 months at $2^{\circ} \mathrm{C}$ in the Minus Eighty Degree Celsius Laboratory Freezer for the ISS (MELFI) unit; while stored in the cold at $2^{\circ} \mathrm{C}$ the 




b)

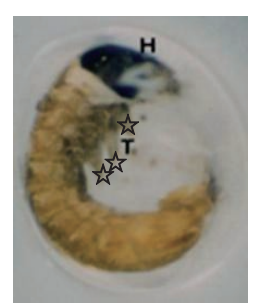

Normal

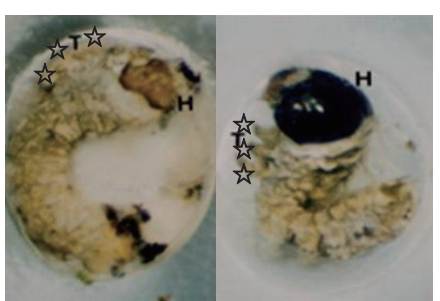

Abnormal
Fig. 3. Effect of microgravity on the embryonic development of eggs of the silkworm, Bombyx mori. a) Percentages of embryos with normal and abnormal embryonic reversal in the unhatched eggs of two ground controls and a flight sample. For one ground control, the eggs were kept continuously at $22.5^{\circ} \mathrm{C}$ in Japan (JPN). Other eggs were divided into two samples, and carried to Kennedy Space Center. One sample was kept continuously at $22.5^{\circ} \mathrm{C}$ as the second ground control (KSC), and the other one was transferred to the Space Shuttle as the flight sample (Flight). After 9 days of space flight, the KSC and flight samples were returned to Japan, and the hatching abilities of all three groups were investigated. Within unhatched eggs, the percentages of embryos with normal (black bars) and abnormal (grey bars) reversal were examined; $n$ is the number of unhatched eggs that were examined. The embryos of normal and abnormal remained alive in the eggs, but the remainder did not reach at the prelarval stage and died. b) Photos of normal (left) and abnormal embryonic reversal (middle and right). T: thoracic, $\mathrm{H}$ : head, Stars mark the position of thoracic legs. The values in parentheses indicate the absorbed dose of radiation that hit each egg, which was estimated from traces of ion particles on CR-39.

embryos in these eggs remain in the diapause-terminated state (Fig. 2B).

Following this, eggs will be transferred to a temperature of $22^{\circ} \mathrm{C}$, where they will start to resume embryogenesis. During this period, blastokinesis or embryonic reversal (Photo $f$ and $g$ in Fig.2B) occurs when embryos turn out their bodies. By monitoring such dynamic changes in embryos, it is possible to determine whether normal embryogenesis proceeds under microgravity conditions. To do this, the eggs will be divided into two groups and held under both $\mu G$ and $1 G$ conditions for a Gravity Contrast Experiment in CBEF. Eggs will be held for 7 days to compare the incidence of the mutation between $1 G$ and microgravity. Some of these eggs will be frozen for analysis of gene expression, and the remainder of the eggs will be transferred to $5^{\circ} \mathrm{C}$ to suppress the proceeding of embryogenesis and hatching in the ISS. These samples will be returned to earth by space shuttle and then transferred to Japan. There, the eggs will be incubated at a warm temperature in the lab to facilitate development and hatching. The hatched larvae will be reared on an artificial diet or mulberry leaves to investigate the above-mentioned items 2, 3 and 4 that are shown in Frame A of Fig.1.

\section{Background which has led to the Space Experiment using the ISS}

The space experiment using the ISS was designed based on the results of previous flight and ground experiments, as described below.

\section{Flight experiment results in STS-84}

An experiment to analyze the effects of microgravity on the embryonic development of silkworms was carried out during a 9 day flight in 1997 aboard the US Space Shuttle Atlantis (STS-84). Prior to the flight, eggs were held at $25^{\circ} \mathrm{C}$ for the first 30 days from oviposition and then continuously $5^{\circ} \mathrm{C}$; under these conditions, diapause terminates at 100 days. The eggs were placed individually into wells at each cross point of 33 straight and vertical ditches for air supply, which were made on egg-hold plates (Polycarbonate, $12 \mathrm{~cm} \times 12 \mathrm{~cm}$ ), so as to hold about 1100 eggs in on plate. The plate was covered with cosmic ray detector film (CR-39) to estimate the absorbed dose of cosmic ray per egg.

Three samples were prepared; one sample was kept at $22.5^{\circ} \mathrm{C}$ in Japan as a ground control (JPN). The other two were carried to the Kennedy Space Center (KSC) where one was maintained as a second ground control, whereas the third was transferred to the Space Shuttle as a flight sample. After the 9 day flight, the two samples taken to KSC were returned to Japan and hatching ability was determined.

The hatching ability of control (JPN) and control (KSC) was $57 \%$ and $44 \%$, respectively, whereas that of the flight sample was substantially lower, just $32 \%$. The low hatching ability of the flight sample may have been caused by exposure to microgravity and/or cosmic rays. An influence of transport to/from Japan could also be involved since the hatching ability of the control (KSC) was lower than that of the control (JPN) sample.

Next, in order to clarify the reason for the high percentage of unhatched eggs in the flight sample, we dissected the unhatched eggs and observed the developmental stage and morphology of the embryos (Fig. 3). We observed two types of embryo; 1) embryos that succeeded in embryonic reversal, developed to the prelarval stage but did not hatch (Left photo in Fig. 3b). In this case, thoracic legs (shown with stars) turned toward the inside after embryonic reversal (blastokinesis) stage; 


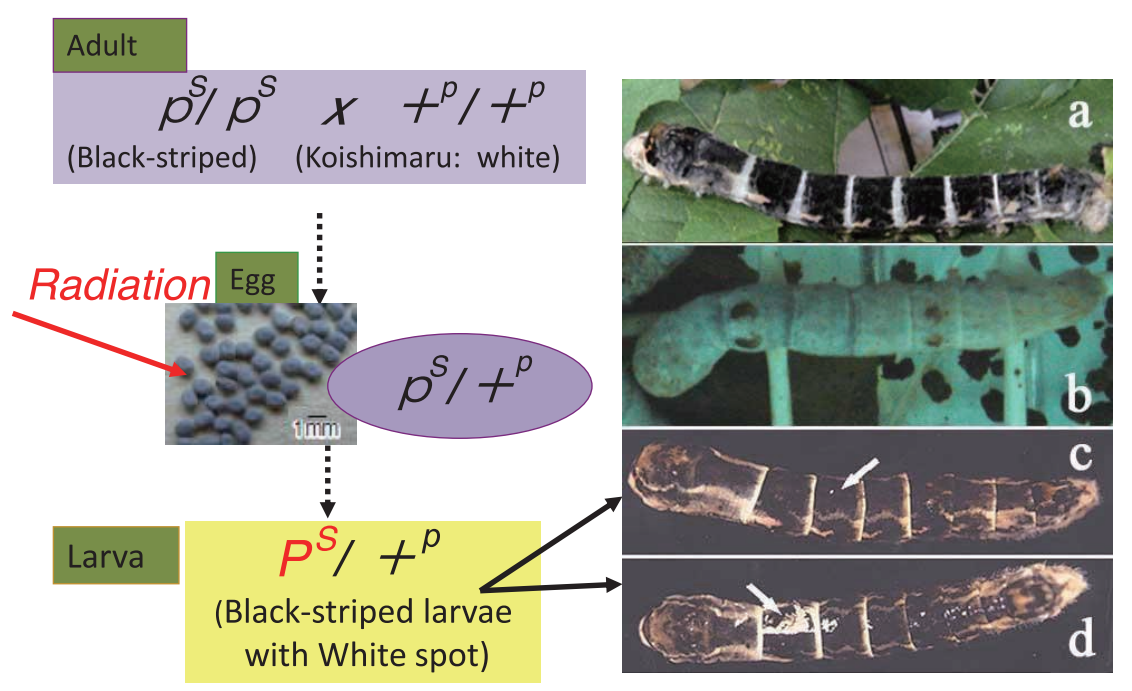

Fig. 4. Detection of somatic mutation. Heterozygous eggs $\left(p^{s} /+^{p}\right)$ are produced by hybridization between black-striped $\left(p^{s} / p^{s}\right)$ (a) and Koishimaru $\left(+^{p} /+^{p}\right)$ (b) strains. When the $p^{s}$ gene of heterozygous eggs is hit by heavy ion particles, white spots appear on the dark brown or dark grey integument on the back of the larvae as shown in photos $c$ and $d$.

this embryo is "Normal", and 2) embryos that could not undertake embryonic reversal, but developed to the prelarval stage; these embryos are named "Abnormal".

The percentage of Normal embryos was $45.2 \%$ and $32.2 \%$ in control (KSC) and flight samples, respectively. By contrast, the corresponding percentage of Abnormal embryos was $20.5 \%$ and $42 \%$, respectively. The embryos of Normal and Abnormal remained alive in the eggs, but the remainder did not reach at the pre-larval stage and died. Since the absorbed dose per egg was no different between embryos showing normal versus abnormal blastokinesis and because the stage of embryonic reversal occurred during the flight, the high abnormal percentage might be caused by microgravity rather than the influence of cosmic radiation.

The results of these experiments gave us important points to consider for the next stage of experiments aboard the ISS. Firstly, we were alerted to the need for management of temperature during the transfer of sample eggs from Japan to KSC. Secondly, we observed the potentially important effect of microgravity. The Space Shuttle has no $1 \mathrm{G}$-generator but the ISS has a $G$ compartment in the CBFS that can control gravity; this stresses the importance of the proposed ISS experiments as the only way to explore the influence of microgravity on the development of silkmoth embryos.

\section{Ground Experiments}

The sensitive response of the 'Black-striped' silkworm strain to heavy ion particles

Our research aims to clarify the correlation between the cumulative dose of cosmic rays and functional alterations to silkworms, as assessed by morphological responses. In order to do this, we have selected a blackstriped strain of silkworm from about 450 stains that are available at the Institute of Genetic Resources, Kyushu University. Exposure of larvae of the black-striped strain of B. mori to $Y$-rays (Nakajima et al., 1972) or neutrons (Machida, 1975) has been shown to induce white spots on the backs of larvae as a somatic mutation. Instead of exposing larvae to $y$-ray irradiation, the eggs were irradiated with heavy ion particles, then hatched and reared and the instances of white spot somatic mutations on black-striped larvae were determined. Fig. 4 shows the principle of the detection of the somatic mutation using the eggs of the black-striped strain.

To use silkworm eggs as a biological model for the detection of cosmic rays, original races of black-striped (Fig. 4a) and Koishimaru (Fig. 4b) was reared. To produce heterozygous eggs of $p^{s} / p$, the female adults of dominant homo-type of $p^{s}$ were mated with male adults of the recessive homo-type Koishimaru $\left(+^{p}\right)$ (Kotani et al., 2002).

When radiation hits a dominant $p^{s}$ heterozygous egg, a few white spots appear on the integument of the blackstriped larvae (Fig. 4c and d). The number of blackstriped larvae with white spots indicates the incidence of somatic mutation by radiation. The incidence depends on the dose and linear energy transfer (LET) of Carbon and Neon (Kotani et al., 2002) (Fig.5). Furthermore, when local hits of heavy ions were directed to the embryo versus to the yolk, the results indicated that somatic mutation was induced only by irradiation of the embryo, not the yolk. Using another approach, we also showed that yolk irradiation caused no bystander effect for somatic mutation (Furusawa et al., 2009), indicating that only irradiation of the embryo by cosmic rays could induce the somatic mutation. Therefore, we propose to have the eggs of the hetero-type stay aboard the ISS for about 3 months (Fig. 2B), and then assess somatic mutations in the larval stage after returning the eggs to earth (Photo $k$ and I in Fig.2). 
a) Carbon

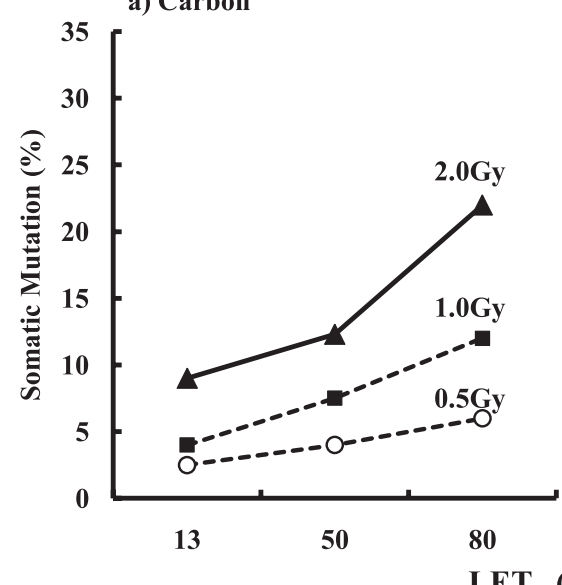

b) Neon

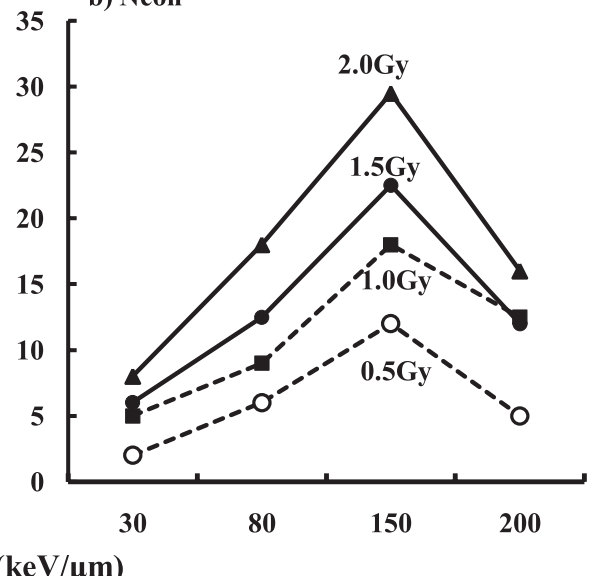

Fig. 5. Dependence of the incidence of somatic mutation on both dose and LET of carbon and neon ion particles. a) Eggs were kept at $25^{\circ} \mathrm{C}$ for the first 2.5 days after oviposition, then transferred to $5^{\circ} \mathrm{C}$. Two months later, the eggs were irradiated with carbon ion particles (290 MeV/u). b) Eggs were kept at $25^{\circ} \mathrm{C}$ for the first 15 days after oviposition, then transferred to $0^{\circ} \mathrm{C}$ to remain in diapause status. These eggs were irradiated using neon ion particles (400 MeV/u).

\section{Sensitivity of diapause eggs and diapause- terminated eggs to heavy ion particles}

Irradiation of diapause eggs by X-rays or heavy ion beams results in somatic mutations that are detectable in $5^{\text {th }}$ instar larvae. In space, physical monitoring of cosmic radiation has detected about $1 \mathrm{mSv}$ per day (Doke et al., 1995). In fact, silkworm diapause eggs on the STS-84 flight received 0.7 1.2 mSv per egg, but this did not result in mutation (Furusawa et al., 1998). Therefore, these results led us to examine the sensitivity of diapause versus diapause-terminated eggs to Neon ion particles (Fig.6). To do this, diapause eggs were held at $25^{\circ} \mathrm{C}$ for 13 days, and then moved to $5^{\circ} \mathrm{C}$. One group of eggs was irradiated with Neon ion particles after 40 days at $5^{\circ} \mathrm{C}$, a time when the eggs are still in diapause. These were then held at $5^{\circ} \mathrm{C}$ for a further 43 days (Fig. $6 a)$. Around this time, the eggs terminate diapause and when moved to a permissive temperature such as $25^{\circ} \mathrm{C}$, the embryos resume development and about 12 days later newly hatched larvae appear. A second group of eggs was treated as above but were exposed to $\mathrm{Ne}$ ion particles after 83 days at $5^{\circ} \mathrm{C}$ when eggs were diapauseterminated (Fig. 6b).

In diapause eggs, the incidence of somatic mutation increased depending on the irradiation dose, and a higher incidence was observed in eggs that were irradiated in
Fig. 6. Sensitivity of diapause eggs and diapause-terminated eggs to $\mathrm{Ne}$ ion particles, and the frequency of somatic mutation in the second generation. Diapause eggs were kept at $25^{\circ} \mathrm{C}$ for the first 13 days after oviposition, and then transferred to $5^{\circ} \mathrm{C}$ for 83 days to terminate the diapause state. Two groups of eggs were irradiated with $\mathrm{Ne}(23 \mathrm{keV} /$ $\mu \mathrm{m}$ ) ion particles at different times. a) After 40 days at $5^{\circ} \mathrm{C}$, one group of eggs was irradiated with $\mathrm{Ne}$ while still in the diapause state and was then held for the remaining 43 days at $5^{\circ} \mathrm{C}$. b) The second group of eggs was irradiated with $\mathrm{Ne}$ at the end of the 83 days at $5^{\circ} \mathrm{C}$ when the eggs were in the diapauseterminated stage. Both groups of eggs were then transferred to $25^{\circ} \mathrm{C}$ to resume embryogenesis. The hatched larvae were reared, and the frequency of somatic mutation was investigated in $5^{\text {th }}$ instar larvae. Female larvae were then grown to adults and mated with male adults of the pe/re strain (egg-color mutation) to examine the effects of radiation on the second generation. The effects were monitored by the number of moths which laid color eggs.

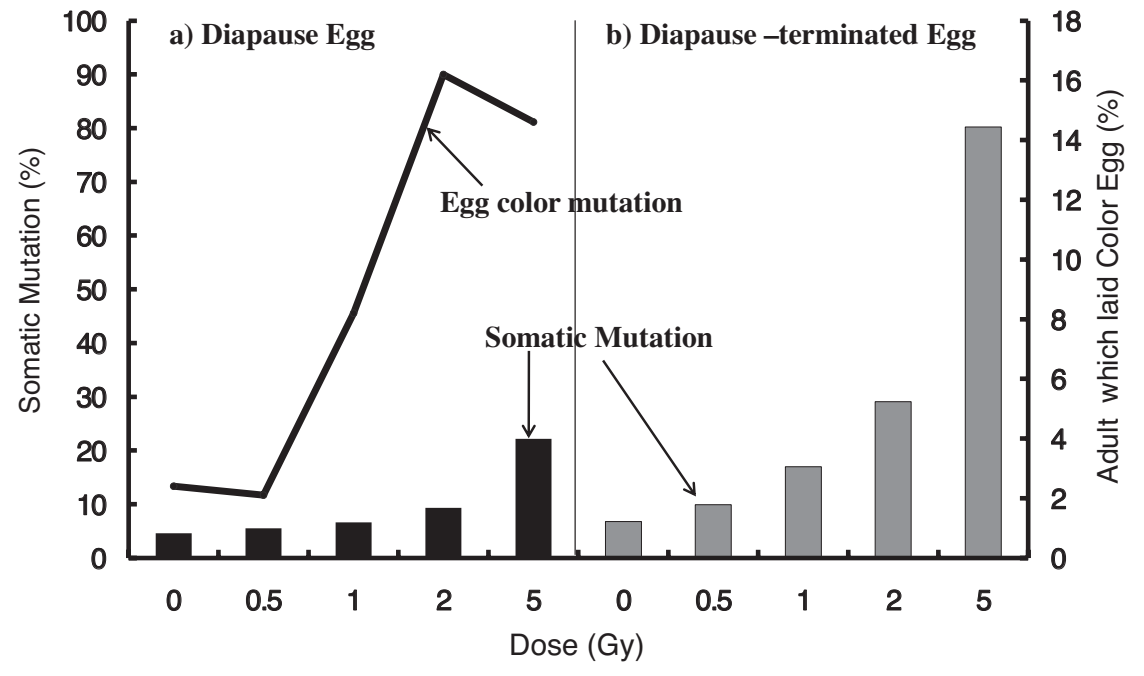

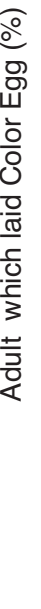




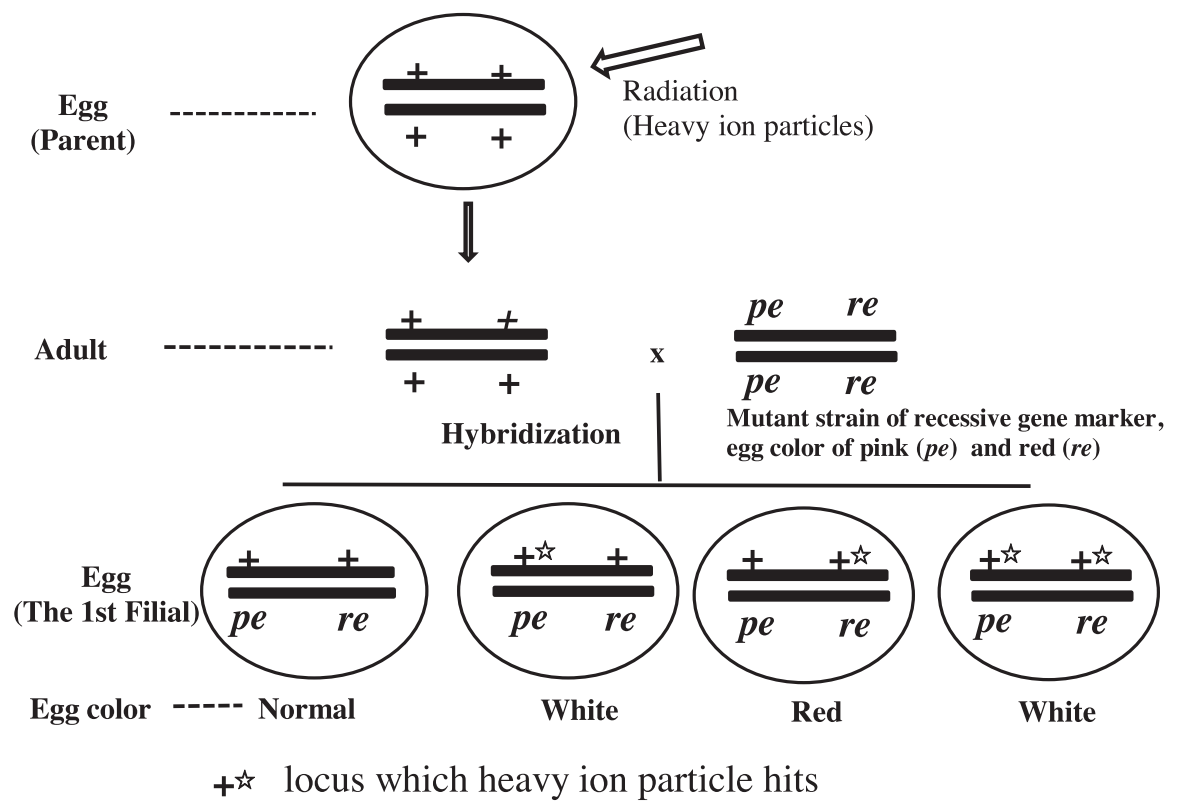

Fig. 7. The principle of the method of specific locus mutation. Eggs of the black-striped strain are exposed to heavy ion particles. The newly hatched larvae are reared to check the incidence of somatic mutation. The larvae with somatic mutations are reared to adults. Female adults are mated with the egg-color mutant strain of recessive gene marker, pink $(p e)$ and red $(r e)$. Thereafter, the colors of the eggs which are laid by the adult female are checked. If $+^{p e}$ was hit by radiation as shown by a star mark ( $\boldsymbol{\zeta}^{\mathrm{r}}$ ), then white eggs will be observed among the laid eggs. If $+{ }^{r e}$ was hit, then red eggs will be observed.

the diapause-terminated state than in the diapause state. The question arose as to whether a radiation effect at the egg stage would be transmitted to the next generation. This was examined using the method of specific locus mutation (Doira, 1978, Tazima, 1978). This method was adopted to detect the frequency of mutation in eggs that were laid by female moths that had been exposed to cosmic radiation as diapause eggs aboard the Russian Space Station Mir (Morimoto and Sakai, 1998).

The principle of this method is described in Figure 7. The eggs of the black-striped strain were exposed to heavy ion particles, and the hatched larvae were reared to adult moths. Female moths were chosen and were hybridized with male moths (w09) with the genotype pe re which were non-irradiated, and then we subsequently observed the color of the eggs laid by the females. If radiation hits the $+{ }^{p e}$ and/or $+{ }^{r e}$ locus (loci) in the embryo of the black-striped strain, eggs of the phenotype pe (white color eggs) and/or re (red color eggs) appear in the first filial generation. As shown by the line graph in Fig. $6 a$, when female $5^{\text {th }}$ instar larvae with white spots were reared to adults and then mated with the male adults of pe/re strain, the number of egg color mutations rose strongly in the next generation. The number of females that laid red color eggs in the next generation increased with increasing dose of irradiation to the egg stage of the female parent.

\section{Sensitivity of 2-day old embryos to a low dose of heavy ion particles}

Next, experiments were conducted to determine which stage of embryo in the egg is the most sensitive to low doses of heavy ion particles. When diapause-terminated eggs were transferred to $25^{\circ} \mathrm{C}$ to resume embryogenesis, the highest sensitivity was detected 2-3 days after the resumption of embryogenesis (data not shown). During silkmoth diapause, the cell cycle is arrested at the $G_{2}$ stage (Nakagaki et al., 1992). Transfer of the eggs from $5^{\circ} \mathrm{C}$ to $25^{\circ} \mathrm{C}$ resulted in active cell division from $G_{2}$ to $M$ phase, which presumably resulted in the high sensitivity. Therefore, the sensitivity of 2-day old embryos after the resumption of embryogenesis to a low dose of $\mathrm{Fe}$ ion particles was tested (Fig. 8). Even a low dose of 0.04 Gy significantly increased somatic mutation compared with non-irradiated eggs. The incidence seemed to increase in a dose dependent manner up to $2.5 \mathrm{~Gy}$, and then reached a maximum level of about $80 \%$ at a dose of 5 Gy. Therefore, when 2-day old embryos receive cosmic rays while at the ISS, we can estimate the dose by using this graph which indicates the relationship between dose and the incidence of somatic mutation at the 5th instar larval stage.

Overall, then, the sequence of the above experiments indicates that we will able to estimate the incidence of somatic mutation that will be induced by cosmic radiation in the space experiment using the ISS.

\section{Our goal for the space experiment}

In space, heavy ion particles are emitted from solar flares and other sources in the galaxy. PADLES (Passive Dosimeter for Life Science Experiments in Space), which consists of thermoluminescent dosimeter (TLD) and cosmic ray detector film (CR-39), is a useful dosimetry technology to estimate the dose or absorbed dose of 


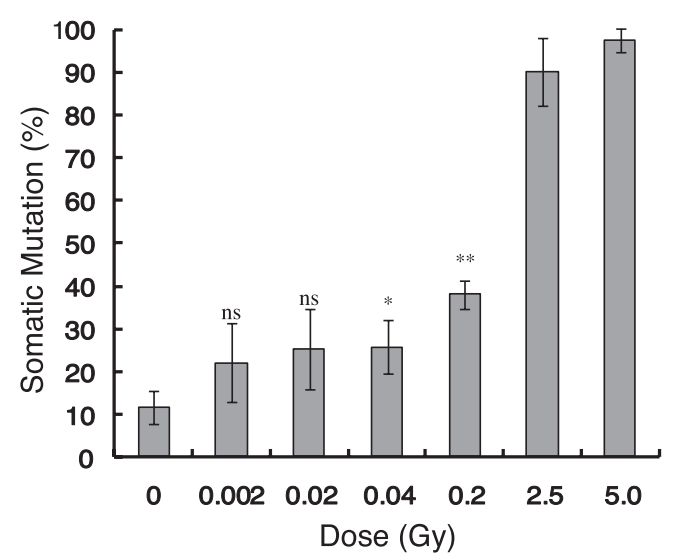

Fig. 8. Sensitivity of 2-day old embryos after the resumption of embryogenesis to a low dose of $\mathrm{Fe}$ ion particles. Diapauseterminated eggs that had been kept at $5^{\circ} \mathrm{C}$ for about 3 months after oviposition were transferred to $25^{\circ} \mathrm{C}$ to resume embryogenesis. On day 2 after the resumption, groups of eggs were irradiated with different doses of $\mathrm{Fe}(200 \mathrm{keV} / \mu \mathrm{m})$ ion particles. Irradiation of eggs with more than $0.04 \mathrm{~Gy}$ of $\mathrm{Fe}$ ions induced a significant incidence of somatic mutation compared to controls ( 0 Gy). Data are the means \pm SEM of $n=3$ independent experiments.

$*: P<0.05, * *: P<0.01 . n s$ indicates not significant.

cosmic rays. However, considering the biological effects of cosmic radiation, we believe that it is important to establish a method of biodosimetry and the proposed project is designed with this goal. The use of the silkworm eggs, which are small and stable, provides a relatively simple measure to study radiation response in a multicellular eukaryotic organism. Therefore, research needs to be done to determine what dose of radiation the eggs can recognize, and what kinds of biological alterations can be used as indicators of cosmic radiation damage.

We also identified several somatic mutation responses to radiation by Carbon, Neon or Ferrous particles in ground experiments (Fig. 4 and 5) and we predict that these will also be excellent, simple markers of radiation exposure in space. These will probably provide highly effective biomarkers of the biological effects of cosmic rays in space experiments using the ISS.

It is not known what dose of cosmic rays induces biological effects that can affect human health during long-term flight. Considering this point, silkworm eggs could be an excellent model for assessing long term radiation exposure in space because the eggs can be maintained in a stable diapause state for least for 2 years at $0^{\circ} \mathrm{C}$, or 3 months at $5^{\circ} \mathrm{C}$ (Furusawa et al., 1982). In the near future (Fig.2), the diapause-terminated eggs will be held on the ISS for about 90 days at $2^{\circ} \mathrm{C}$, and subsequently for 7 days at $20^{\circ} \mathrm{C}$, and then hatched and assessed for damage or mutation. What dose of cosmic rays do embryos receive?

The dose of cosmic rays as measured by physical monitoring is about $1 \mathrm{mSv}$ per day (Doke et al., 1995).
Recently, the absorbed dose in the ISS was estimated to be about $0.5 \mathrm{mSv}$ (Cucinotta et al., 2008). The homepage of JAXA (http://idb.exst.jaxa.jp/ db_data/padles/ S002. php) states that the absorbed dose is $0.28 \mathrm{mGy} /$ day. In space, the diapause-terminated eggs will be receiving about $0.28 \mathrm{mGy} /$ day over a period of about 90 days. Therefore, these eggs will receive a cumulative dose of about 0.025 Gy. Our data from ground experiments showed that $0.04 \sim 0.2$ Gy of Fe ion particles induced somatic mutations in about $25-40 \%$ of animals, which was significantly different from the incidence of mutations in controls (Fig. 8). Hence, we predict that the cumulative dose of cosmic rays over the 90 days flight should induce somatic mutations that we can assess and quantify.

It will be interesting to determine the molecular basis of mutations induced by cosmic radiation. Unfortunately, the nucleotide sequence of the $p^{s}$ gene has not yet been identified. Alternative genes, NAD-sorbitol dehydrogenase (Sakano et al., 2004) and trehalase (Huang et al., 2005), whose sequences are known, are potential candidates to use instead to survey the effects of cosmic radiation, because they are expressed at specific times during embryonic development. In addition, the homologue of the p53 gene (tumor suppressor gene) was recently identified in silkworm eggs (Nagaoka et al., unpublished). The expression of mammalian $p 53$ has been reported in relation to the space environment (Ohnishi et al., 1996, $1999,2000)$. Thus, eggs radiated on ISS will be used to analyze the expression pattern of p53 homologue gene. From comparative analysis of the silkworm and mammal, role of p53 gene function under the space environment can be elucidated.

In experiments with the whole body of Drosophila melanogaster (Inoguchi and Ikenaga, 2000) and the eggs of Carasius morosus (Bucker et al., 1986), synergistic effects of radiation and microgravity on the incidence of mutation were reported, but such a phenomenon was not seen in experiments with human, mouse or hamster cell lines (Ikenaga et al., 2002). Therefore, to assess this contradiction, experiments will be done using the 2-day old eggs after resumption of embryogenesis (the stage that is the most sensitive to radiation) to compare $\mu G$ to $1 G$ exposures using the $1 G$ compartment on the ISS. Related to this experiment, we will confirm whether normal embryonic development proceeds under microgravity.

Through this research, the authors strongly expect to establish a biodosimetry method using silkworm eggs, and thus contribute to the protection of human health from cosmic radiation during long-term stays in space.

\section{Acknowledgements}

The authors are greatly indebted to Professor Kenneth B. Storey and Mrs. Janet M. Storey (Carleton University, Ottawa, Canada) for their advice and critical corrections of the manuscript. This work was partly funded by the "Ground Research Announcement for Space Utilization" promoted by the Japan Space Forum and NASDA. 


\section{References}

Bucker, H., Facius, R., Horneck, G., Reitz, G., Graul, E., Berger, H., Hoffken, H., Ruther, W., Heinrich, W., Beaujean, R. and Enge, W. (1986) Embryogenesis and organogenesis of Carausius morosus under spaceflight conditions, Adv. Space Res., 6, 115-124.

Cucinotta, F.A., Kim, M. H., Willingham, V. and George, K. A. (2008) Physical and biological organ dosimetry analysis for international space station astronauts, Radiat. Res., 170, 127-138.

Doira, H. (1978) Genetic stocks of the silkworm. In: The silkworm: an important laboratory tool. Ed. Tazima, Y., pp.53-81, Kodansha (Tokyo).

Doke, T., Hayashi, T., Kikuchi, J., Haseb, N., Nagaoka, S., Kato, M. and Badhwar, G.D. (1995) Real time measurement of LET distribution in the IML-2 Space Lab (STS-65), Nucl. Instr. Meth. Phys. Res., A365, 524.

Furusawa, T., Shikata, M. and Yamashita, O. (1982) Temperature dependent sorbitol utilization in diapause eggs of the silkworm, Bombyx mori, J. Comp. Physiol., $147,21-26$

Furusawa, T, Kotani, E., Ichida, M., Sugimura, Y., Yamanaka,H., Takahashi, S., Fukui, M., Kogure, K., Sakaguchi, B., Fujii, H., Ikenaga, M. and Watanabe, T. (1988) Embryonic development in the eggs of the silkworm, Bombyx mori, exposed to the space environment, J. Jpn. Soc. Microgravity Appl., 15 Supplement II, 666-671.

Furusawa, T., Fukamoto, K., Sakashita, T., Suzuki, E., Kakizawa, T., Hamada, N., Funayama, T., Suzuki, H., Ishioka, N., Wada, S., Kobayashi, Y. and Nagaoka, S.(2009) Targeted heavy-ion microbeam irradiation of the embryo but not yolk in the diapause egg of the silkworm, Bombyx mori, induces the somatic mutation, J. Radiat. Res., 50, 371-375.

Huang, J., Arimatsu, Y., Sadakane, K., Endo, Y. and Furusawa, T. (2005) Gene expression and localization of trehalase during embryonic development of the silkworm, Bombyx mori, Int. J. Wild Silkmoth \& Silk, 10, 17-26.

Ikenaga, M., Hirayama, J., Kato, T., Kitao, H., Han, Z., Ishizaki, K., Nishizawa, K., Suzuki, F., Cannon, T.F., Fukui, K., Shimazu, T., Kamigaichi, S. Ishioka, N. and Matsumiya, H. (2002) Effect of space flight on the frequency of micronuclei and expression of stressresponsive proteins in cultured mammalian cells, J. Radiat. Res., 43 Suppl., S141-S147

Inoguchi, M. and Ikenaga, M. (2000) Cosmic Irradiation. In: Inoguchi, H, Okada, M. Kuchitsu, K. and Kobayashi, S. (Eds.), Science for Space Utilization Research. Shokabo, Tokyo, pp.235-254.

Kotani, E., Furusawa, T., Nagaoka, S., Nojima, K. Fujii, H., Sugimura, Y., Ichida, M., Suzuki, E., Nagamatsu, A., Todo, T. and Ikenaga, M. (2002) Somatic mutation in larvae of the silkworm, Bombyx mori, induced by heavy ion irradiation to diapause eggs, J. Radiat. Res., 43 Suppl., S193-S198.
Machida, I. (1975) A comparison of mutagenic effects by $2 \mathrm{MeV}$ neutrons and $200 \mathrm{kVp}$ X-rays in prophase I oocytes of the silkworm, Bombyx mori L., J. Sericult. Sci. Japan, 44, 11-16.

Morimoto, K. and Sakai, A. (1998) Long-term effect of cosmic environment on the diapausing eggs of the silkworm, Bombyx mori. Rept. on the Results of Space Experiments. NASDA. IV105-115.

Nakagaki, M., Takei, R., Nagashima, E. and Yaginuma, T. (1992) Cell cycles in embryos of the silkworm, Bombyx mori, Roux's Arch. Dev. Biol., 200, 223-229.

Nakajima, M., Takimoto, T. and Miyakoda, T. (1972) Radiation effects on silkworm, Bombyx mori L., with special reference to changes in the frequencies of induced somatic mutations during different stages of larval development, J. Sericult. Sci. Japan, 41, 359364.

Ohnishi, T., Inoue, N., Matsumoto, H., Ohnishi, K., Fukuda, S. and Nagaoka, S. (1996) Cellular content of p53 protein in rat skin after exposure to a space environment, J. Appl. Physiol., 81, 183-185.

Ohnishi, T., Takahashi, A., Wang, X., Ohnishi, K., Ohhira, Y. and Nagaoka, S. (1999) Accumulation of tumor suppressor p53 in rat muscle during a space flight, Mutat. Res., 430, 271-274.

Ohnishi, T., Wang, X., Fukuda, S., Takahashi, A., Ohnishi, K. and Nagaoka, S. (2000) Accumulation of tumor suppressor p53 in rat muscle after a space flight, $A d v$. Space Res., 25, 2119-2122.

Sakano, D., Furusawa, T., Sugimura, Y., Storey, J.M. and Storey, K.B. (2004) Metabolic shift in carbohydrate metabolism during embryonic development of nondiapause eggs of the silkworm, Bombyx mori, J. Insect Biotech. Sericol., 73, 15-22.

Shimada, T., Ebinuma, H. and Kobayashi, M. (1986) Expression of homeotic genes in Bombyx mori estimated from asymmetry of dorsal closure in mutant/ normal mosaics, J. Exp. Zool., 240, 335-342.

Takami, T. and Kitazawa, T. (1960) Embryonic stage of the silkworm, Bombyx mori, Bull. Sericulture Experiment Station, 75, 1-3.

Tajima, Y. (1978) Radiation mutagenesis of the silkworm. In 'The silkworm: an important laboratory tool (ed. Tazima, Y.)', Kodansha (Tokyo) pp.213-245. 
Space Experiment using Bombyx eggs

- 70 - 